\title{
Direct Evidence for Dynamic Phase Transformation during High Temperature Deformation in Ti-64
}

\author{
Christopher S. Daniel ${ }^{1}$, Chi-Toan Nguyen ${ }^{1}$, Michael D. Atkinson ${ }^{1}$, João Quinta da Fonseca ${ }^{1}$
}

${ }^{1}$ Centre for Light Alloy Research and Innovation, University of Manchester, Manchester, M13 9PL, UK

\begin{abstract}
The microstructure of Ti-64 strongly affects the forming and in-service behaviour of aerospace components. The development of microstructure, including crystallographic texture, during thermomechanical processing involves changes caused by deformation, annealing and phase transformation. Recently, it has been reported that deformation and transformation occur concurrently, in a dynamic $\alpha \rightarrow \beta$ transformation process, driven by the net flow softening it produces. Here, we present the results of an in-situ synchrotron diffraction experiment that aimed to detect and measure the extent of dynamic phase transformation during hot deformation and its contribution to the crystallographic texture of the alloy. Forged Ti- 64 samples were deformed in uniaxial tension at $950^{\circ} \mathrm{C}$ and a strain rate of about $0.02 \mathrm{~s}^{-1}$, using an electro-thermal mechanical tester (ETMT) mounted on the I12: JEEP beamline at Diamond Light Source. By using a high energy 89 $\mathrm{keV}$ synchrotron X-ray beam and a fast acquisition rate $(10 \mathrm{~Hz})$ we were able to measure, during deformation, the internal elastic lattice strains in the two phases, the crystallographic texture of both phases and the $\alpha \rightarrow \beta$ phase fraction. The results show, for the first time, direct evidence of dynamic transformation during isothermal hot deformation.
\end{abstract}

\section{$\underline{\text { Keywords }}$ \\ alpha beta processing; synchrotron diffraction; ti64}

\section{Introduction}

The properties and performance of dual-phase Ti alloys in-service are strongly influenced by their microstructure and crystallographic texture, which can be controlled by thermomechanical processing during manufacture. However, it is difficult to predict how the two-phase microstructure evolves during hotdeformation as it depends on complex interactions between the $\alpha$ (hexagonal close-packed, hcp) and $\beta$ (body-centred-cubic, bcc) phases. Recently, post-mortem microstructural observations of hot-deformed Ti-64 samples have shown evidence that this interaction involves dynamic $\alpha \rightarrow \beta$ transformation, during deformation, which increases the volume fraction of $\beta$-phase by up to $12 \%$ [1,2]. In steels, dynamic transformation is a well-accepted mechanism, in which continuous plastic deformation transforms the austenite to ferrite, controlling grain refinement [3]. Nevertheless, dynamic transformation is missing from any efforts to model the hot deformation behaviour of dual-phase Ti alloys.

The aim of the work presented here was to use high energy synchrotron X-ray diffraction (SXRD) to observe dynamic transformation in Ti-64 directly, during isothermal deformation at $950^{\circ} \mathrm{C}$, high in the $\alpha+\beta$ regime. At these temperatures, high strain rates of deformation are required to develop the conditions for dynamic phase transformation. Therefore, only synchrotron radiation can be used for in-situ observations [4,5], which has significantly faster measurement times than neutron diffraction [6].

The setup presented here makes use of an electro-thermal mechanical tester (ETMT) mounted on Diamond's I12 synchrotron beamline [7], to provide fast isothermal deformation in tension, at a strain rate of about $0.02 \mathrm{~s}^{-1}$, to a matchstick Ti-64 sample. Deforming in tension minimises beam attenuation - compared with uniaxial compression which leads to material spread in the beam direction - meaning the $\alpha$ and $\beta$ peak profiles remain sharp, with little overlap, throughout the test. A fast detector frame rate, recording at $10 \mathrm{~Hz}$ acquisition frequency, is able to capture a highly resolved dataset of diffraction pattern images, containing information on the two-phase elastic lattice strain, grain reorientation and volume fraction as the high temperature microstructure evolves. Changes in the bulk $\alpha / \beta$ volume fraction were obtained following full azimuthal integration of the diffraction pattern images and Rietveld refinement in TOPAS, whilst the transformation response of the different $\alpha$ and $\beta$ grain orientations were monitored via intensity changes using single peak profile fitting.

\section{Experimental Methodology}

\subsection{Starting Material}

Ti-64 samples were taken from a unidirectional forged plate and solution heat-treated at $950^{\circ} \mathrm{C}$, followed by $1{ }^{\circ} \mathrm{C} / \mathrm{min}$ cooling. The cooling rate was chosen to produce a fine equiaxed grain structure, with a diameter of between $1 \mu \mathrm{m}$ and $20 \mu \mathrm{m}$. For dislocation free grains, the material was then annealed at $500^{\circ} \mathrm{C}$ for 48 hours, with the resulting microstructure shown in Figure 1 a). A matchstick sample was then machined with dimensions $2 \mathrm{~mm} \times 2 \mathrm{~mm} \times 55 \mathrm{~mm}$. 

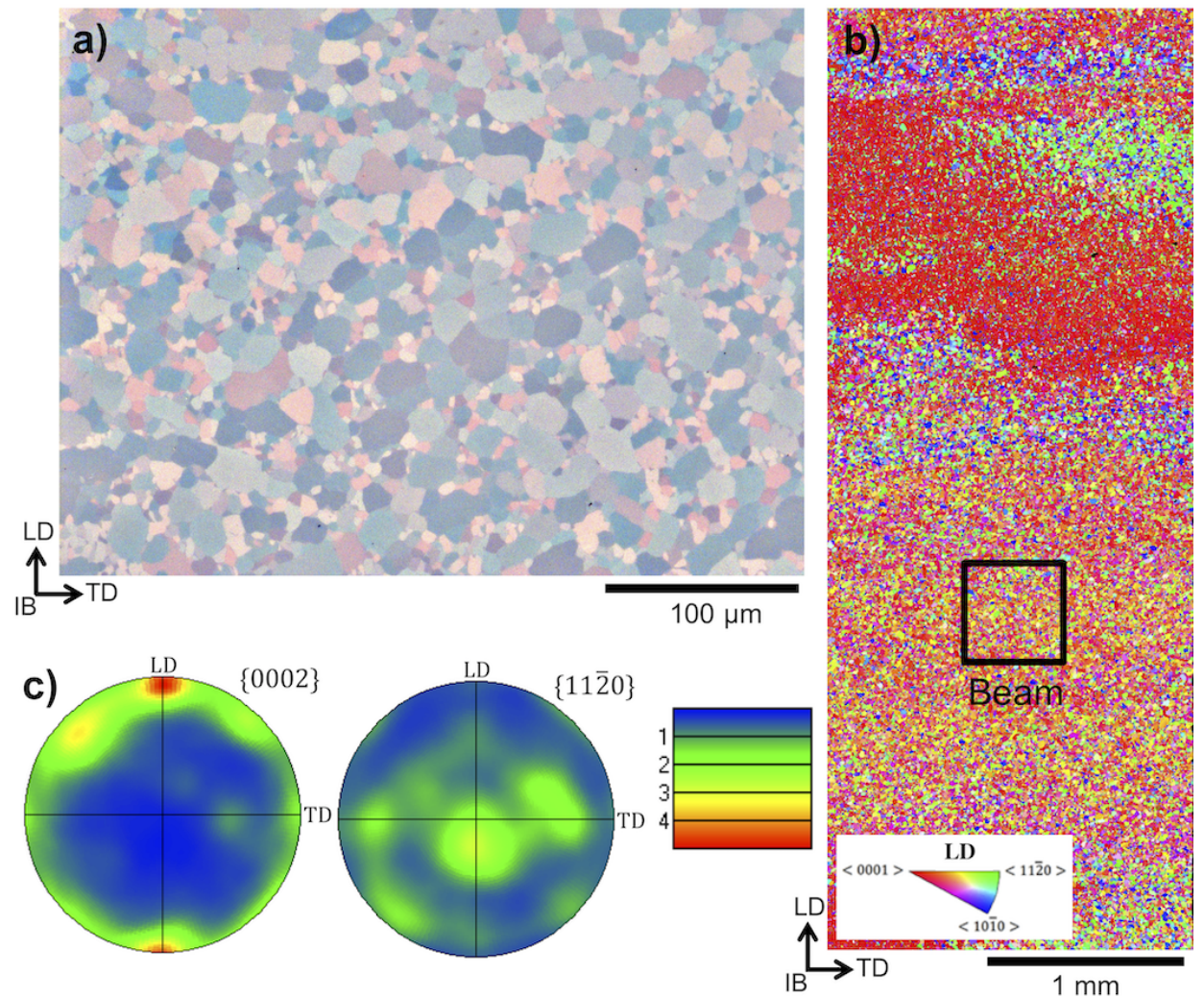

Figure 1: The starting Ti-64 alloy contains many fine equiaxed grain structures in (a), taken using polarised light. The distribution of $\alpha$-orientations in (b) are shown along the cross-section of the matchstick sample, along with a representation of the beam area used for the synchrotron analysis. The crystallographic texture represented in (c) favours 0002|| $\mathrm{LD}_{\text {(loading direction) and }} 11 \overline{2} 0|| \mathrm{IB}$ (in-beam direction).

To characterise the starting orientations within the chosen specimen electron backscatter diffraction (EBSD) was used. EBSD analysis was performed using the TESCAN MIRA3 field emission gun scanning electron microscope (FEG-SEM), equipped with an AZtecHKL system for pattern indexing. EBSD data was collected at an operating voltage of $20 \mathrm{kV}$ with a working distance of $\sim 20 \mathrm{~mm}$, using a $1 \mu \mathrm{m}$ step size covering an area $\sim 14 \mathrm{~mm}{ }^{2}$. The $\alpha$-phase orientation map and accompanying 0002 and $11 \overline{2} 0$ pole figures are shown in Figure 1b) and c), respectively.

The crystallographic texture shows the basal pole maxima of the plate is aligned with a longitudinal loading direction (LD) of the matchstick sample. The crystallographic texture also shows prismatic $11 \overline{2} 0$ maxima in the compression direction of the plate, aligned with the in-beam direction (IB) of the synchrotron experiment, along with a weaker 1010 formed in the transverse direction (TD). The initial forging process is also seen to produce a micro-texture that is relatively inhomogeneous, with macrozones clearly visible across the sample, in Figure 1b).

\subsection{Electro-Thermal Mechanical Tester (ETMT)}

The Ti-64 sample was heated and deformed using an electro-thermal mechanical tester (ETMT). The sample gauge length on the ETMT was set at 30 mm, which maximises the distance between the water-cooled grips, increasing the length of the central hot-spot. An R-type thermocouple (99.99\% Pt and 87:13 wt.\% Pt/Rh) was spot-welded at the centre of the specimen, providing feedback for direct resistive heating in a temperature control mode. Heating was applied at a rate of

$5^{\circ} \mathrm{C} \mathrm{s}^{-1}$ to $950^{\circ} \mathrm{C}$ and held for 2 minutes. The ETMT was initially controlled under load control, enabling free thermal expansion of the sample without significant stress accumulation [8]. Then, deformation was applied in tension, using the crosshead position control, at a rate of $0.15 \mathrm{~mm} \mathrm{~s} \mathrm{~s}^{-1}$ to $3 \mathrm{~mm}$. This corresponds to a strain rate at the centre of the sample of about $0.02 \mathrm{~s}^{-1}$ up to a true strain of 0.5 , measured using a resistance method, whilst temperature control maintains a $950^{\circ} \mathrm{C}$ deformation temperature to within $\pm 5^{\circ} \mathrm{C}$. Resistance was measured across the central region of the sample by attaching two Pt resistivity wires, 2 mm apart, either side of the thermocouple, providing an alternative measurement of true strain accounting for changes in the cross-sectional area [9]. The resistance increase during deformation in tension, $R_{\mathrm{t}}$, is compared to the resistance before applying load, $R_{\mathrm{s}}$, to calculate a true plastic strain, $\varepsilon$, given by [9];

$$
\varepsilon=\ln \sqrt{\frac{R_{\mathrm{t}}}{R_{\mathrm{s}}}}
$$

Equation 1

\subsection{High Temperature In-Situ Synchrotron X-ray Diffraction (SXRD)}

The ETMT was mounted on the I12:JEEP (Joint Engineering, Environmental and Processing) beamline at Diamond Light Source, allowing for simultaneous recording of diffraction pattern rings in transmission mode during the thermomechanical test. A synchrotron X-ray beam energy of 89 keV (wavelength, $\lambda=0.1392 \AA$ ), with slits adjusted $0.5 \mathrm{~mm} \times 0.5 \mathrm{~mm}$, was selected to illuminate the sample. A 2D Pixium detector was positioned at a sample-detector distance of $1200 \mathrm{~mm}$, acquiring data at a frequency of $10 \mathrm{~Hz}$, able to measure the high strain rate deformation micromechanics and increase sampling in the linear elastic regime. To ensure sufficient signal at this fast frame rate, binning of $2 \times 2$ pixels was applied at the detector during acquisition, with a pixel size set to $0.296 \mu \mathrm{m} \times$ $0.296 \mu \mathrm{m}$ on the detector.

\subsection{SXRD Data Analysis}

The 2D diffraction images were integrated using DAWN (Data Analysis WorkbeNch), to obtain plots of intensity versus two-theta, using slices of $10^{\circ}$. To increase the density of points and improve the fitting procedure, six points at each two-theta increment were included in the analysis; using three slices of $10^{\circ}$ from the top and bottom of the diffraction pattern, with a total azimuthal range of $30^{\circ}$ around the loading axis. 
The diffraction peaks were fitted in Python, using a pseudo-Voigt function from the lmfit package [10]. Monitoring changes in the peak profiles provides information on the response of the two-phases during high temperature loading. The amplitude of the peaks is of particular importance, since it corresponds to the volume fraction of grains orientated in a given direction. The change in the position of each diffraction peak can also be used to calculate the volume averaged elastic strain, although results for the microstrain response are not presented here and will be published elsewhere.

To measure average, bulk changes in volume fraction and unit cell parameters of both phases, Rietveld refinement of the full peak profile was also conducted in TOPAS, following an entire azimuthal integration of the diffraction images.

For quantitative texture evolution, a least-squares Rietveld refinement was carried out using the software MAUD (Materials Analysis Using Diffraction) at different stages of the thermomechanical test. Crystallographic texture was calculated by determining the full orientation distribution function (ODF), using an extended version of the WIMV (Williams-Imhof-Matthies-Vinel) algorithm [11], with the results to be published elsewhere in the near future.

\section{Results}

Images of the sample before and after deformation are shown in Figure 2, along with the recorded true stress versus true strain response of the material, where points along the flow stress curve represent the diffraction image sampling rate.


Figure 2: The sample geometry before and after high temperature loading in the electro-thermal mechanical tester $($ ETMT) in $($ a), where a $0.5 \times 0.5$ mm synchrotron beam is initially placed slightly above the thermocouple. The true stress $(\sigma)$ versus true strain $(\varepsilon)$ response in (b) shows characteristic flow softening after yield, with each measurement point corresponding to a diffraction image collected at $10 \mathrm{~Hz}$ acquisition frequency.

An example of the two-phase diffraction pattern rings at $950^{\circ} \mathrm{C}$ is shown in Figure 3 , prior to deformation. The $\alpha$ and $\beta$ intensity versus two-theta profiles are obtained following caking of the $2 \mathrm{D}$ diffraction image, in this case taken with respect to the loading direction. An example fit to the peak profiles is also shown in Figure 3, for the 0002, 110 and $10 \overline{1} 1$ peaks.
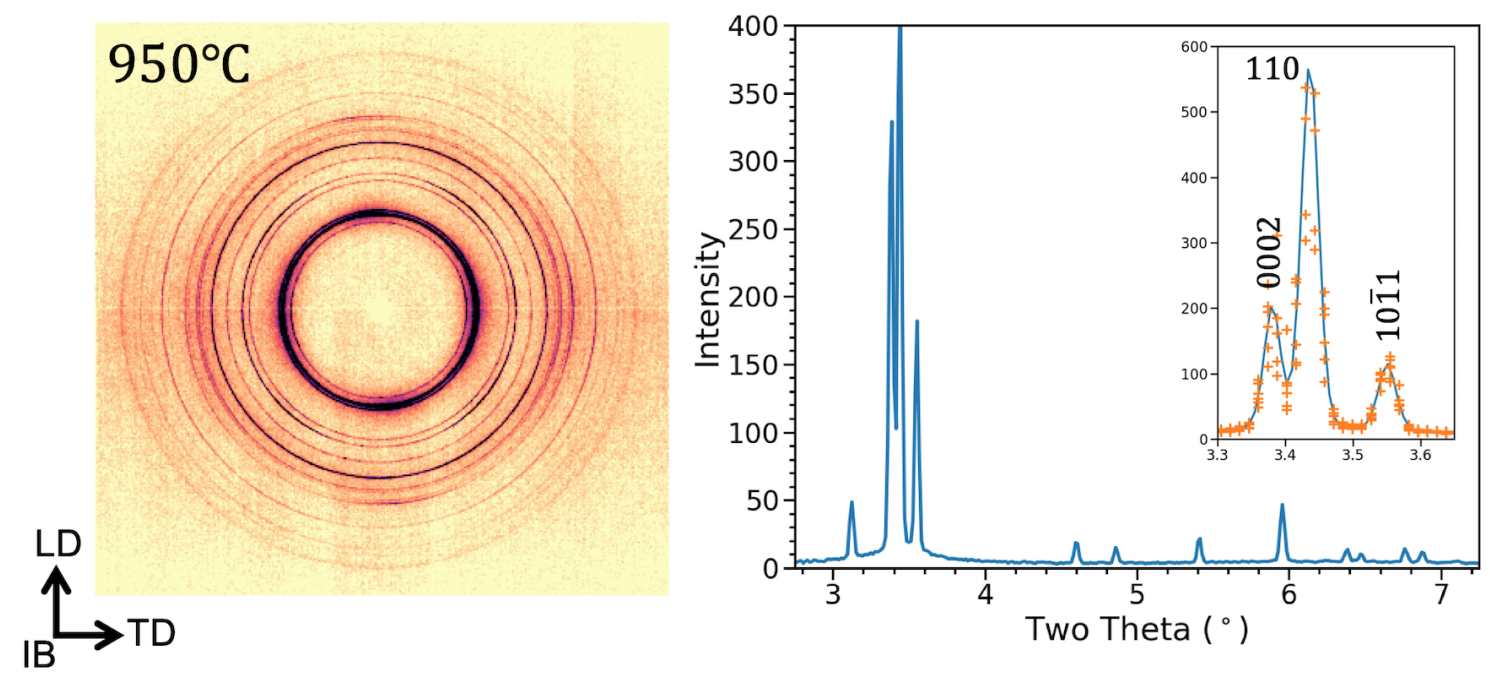

Figure 3: Diffraction pattern rings at $950^{\circ} \mathrm{C}$, showing the separation of the $\alpha$ and $\beta$ lattice plane peaks (left). Caking of the data produces two-dimensional intensity versus two-theta plots (right), with peak profile analysis used to discern the behaviour of the different grain orientations during high temperature

deformation, along with an example fit shown for the $\alpha 0002, \beta 110$ and $\alpha 10 \overline{1} 1$ peaks. A full azimuthal cake of the data can also be used to monitor

changes in the $\alpha / \beta$ volume fraction, based on intensity of the $\alpha$ and $\beta$ peaks. In addition, intensity variations around the rings for each of the lattice plane peaks can be used to determine crystallographic texture. 


\subsection{Dynamic Transformation}

Full pattern analysis using TOPAS, following the $2 \pi$ azimuthal integration of the rings, reveals that the overall $\beta$ volume fraction of the material increases during high temperature deformation. The changes in phase volume fraction are shown in Figure 4 . Initially, a stable $\alpha / \beta$ volume fraction of $28: 72$ is reached during the high temperature hold. Then, during deformation, an $\alpha \rightarrow \beta$ transformation occurs, with the volume fraction of $\beta$-phase increasing by $\sim 5 \%$ up to 0.2 strain. Monitoring the changes in the $\alpha_{\mathrm{c}}, \alpha_{\mathrm{a}}$ and $\beta_{\mathrm{a}}$ lattice parameter diffraction results confirms the that temperature remains constant during this change, at least up to 0.2 strain.
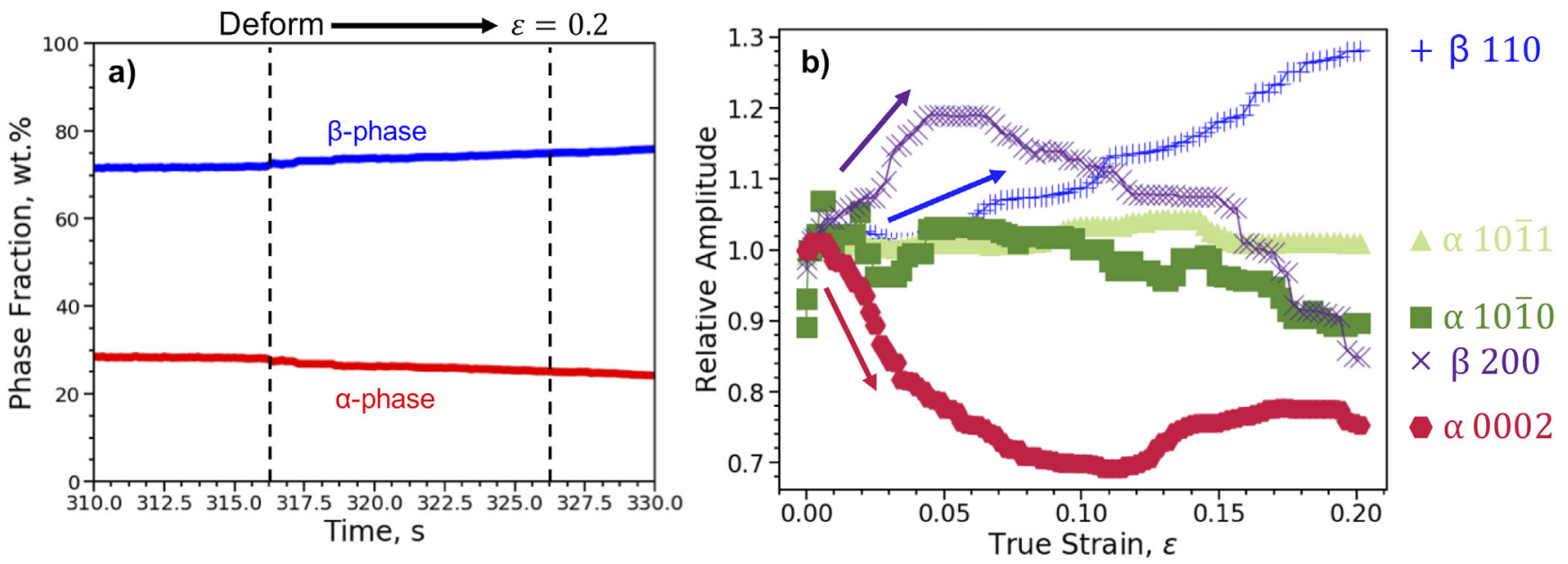

Figure 4: Changes in the $\alpha$ and $\beta$ volume fraction throughout the entire thermomechanical cycle were calculated using Rietveld refinement in TOPAS. The $\beta$ phase fraction increases and the $\alpha$ phase fraction decreases during deformation at high temperature up to 0.2 strain in (a). Changes in the intensities of individual $\alpha$ and $\beta$ lattice plane peaks, with respect to the loading axis, reveal different grain behaviours during deformation in (b). The $\alpha 10 \overline{1} 0$ and $10 \overline{1} 1$ remain relatively constant with increasing strain. The $\alpha 0002$ intensity drops sharply during high temperature deformation. The fall in $\alpha$ peak intensity is mirrored by amplitude increases in both the $\beta 200$ and 110 peaks; direct evidence for dynamic $\alpha \rightarrow \beta$ transformation.

Single peak profile analysis shows the $\alpha$-grains that undergo dynamic transformation during deformation have a particular orientation with respect to the loading direction. A plot of the relative change in amplitude of the peak profiles for each of the $\alpha$ and $\beta$ lattice planes is shown in Figure 4 . Up to 0.2 applied strain, the amplitude of the $\alpha 1010$ and 1011 lattice planes remain relatively unchanged. However, the amplitude of the $\alpha 0002$ peak decreases sharply during the test. At the same time, the amplitude of both the $\beta 110$ and 200 peaks increase significantly, as the volume fraction of $\beta$ increases, mirroring the behaviour of the $\alpha 0002$ peak.

\section{Discussion}

Measurement of the bulk $\alpha / \beta$ volume fraction, as well as the intensity measurements of the individual lattice plane peaks, show an increase in $\beta$-phase during high temperature deformation, which is clear evidence of a dynamic transformation mechanism. In previous ex-situ studies, the effect has been thought to contribute to a $5 \%$ increase in $\beta$ phase fraction at a strain of 1.0 and up to as much as $12 \%$ at a strain of 4.0 [1]. Here, our in-situ studies confirm that dynamic transformation is active at lower strains, up to 0.2 , and that it is more prevalent than first thought.

The contribution of dynamic transformation to the deformation behaviour is most significant when considering the changes of the grains with basal poles orientated in the loading direction. The sharp decrease in amplitude of the $\alpha 0002$ peak suggests the grains with this orientation rapidly undergo transformation. Slip can also contribute to a reduction in peak intensity through reorientation [4], although changes via slip are only ever gradual and the diffraction ring profile showed little evidence for traces of grain rotation. The fact that the amplitude of both the $\beta 110$ and 200 peaks also increase at the same time, during deformation, shows a close transformation relationship exists between the two phases.

Since the transformation behaviour of the $\alpha$-grains is related to their orientation with respect to the loading direction, the mechanism could also explain the crystallographic texture evolution produced during $\alpha-\beta$ processing of these dual-phase Ti alloys. Dynamic transformation is thought to be mechanically activated and driven by the net flow softening it produces [2], favouring microstructure evolution towards a softer $\beta$-phase. However, this explanation does not consider that the strength of the $\alpha$-phase depends on its crystallographic orientation. The favoured transformation of 'harder' $\alpha$ grain orientations, with the most stress accumulation, would contribute most significantly to this softening behaviour.

\section{Conclusions}

In-situ synchrotron measurements have provided compelling direct evidence of dynamic transformation in Ti-64 during isothermal deformation. The $\beta$ phase fraction increased by $5 \%$ after a true strain of 0.2 at $950^{\circ} \mathrm{C}$. Single peak profile analysis reveals that dynamic transformation also preferentially consumes particular $\alpha$ variants at high temperature, with the mechanism favouring transformation of the $\alpha$ grains with [0002] aligned in the loading direction. The results also imply that dynamic transformation will affect the texture development in Ti-64, which has implications for the processing of formed aerospace components.

\section{Acknowledgements}

This work was funded by LightForm, a UK Engineering and Physical Sciences Research Council (EPSRC) programme grant (EP/R001715/1).

\section{References}

[1] B. Guo, S.L. Semiatin, J.J. Jonas, S. Yue, Dynamic transformation of Ti-6Al-4V during torsion in the two-phase region, J. Mater. Sci. 53 (2018) 9305-9315. doi:10.1007/s10853-018-2237-

[2] B. Guo, S.L. Semiatin, J. Liang, B. Sun, J.J. Jonas, Opposing and Driving Forces Associated with the Dynamic Transformation of Ti-6Al-4V, Metall. Mater. Trans. A Phys. Metall. Mater. Sci. 49 (2018) 1-5. doi:10.1007/s11661-018-4551-1.

[3] L. Zhao, N. Park, Y. Tian, A. Shibata, N. Tsuji, Combination of dynamic transformation and dynamic recrystallization for realizing ultrafine-grained steels with superior mechanical properties, Sci. Rep. 6 (2016) 1-11. doi:10.1038/srep39127. 
[4] D. Canelo-Yubero, G. Requena, F. Sket, C. Poletti, F. Warchomicka, J. Daniels, N. Schell, A. Stark, Load partition and microstructural evolution during in situ hot deformation of Ti-6Al-6V2Sn alloys, Mater. Sci. Eng. A. 657 (2016) 244-258. doi:10.1016/j.msea.2016.01.059.

[5] A. Stark, M. Rackel, A. Tchouaha Tankoua, M. Oehring, N. Schell, L. Lottermoser, A. Schreyer, F. Pyczak, In Situ High-Energy X-ray Diffraction during Hot-Forming of a Multiphase TiAl Alloy, Metals (Basel). 5 (2015) 2252-2265. doi:10.3390/met5042252.

[6] G.C. Obasi, R.J. Moat, D.G. Leo Prakash, W. Kockelmann, J. Quinta da Fonseca, M. Preuss, In situ neutron diffraction study of texture evolution and variant selection during the $\alpha \rightarrow \beta \rightarrow \alpha$ phase transformation in Ti-6Al-4V, Acta Mater. 60 (2012) 7169-7182. doi:10.1016/j.actamat.2012.09.026.

[7] J. Romero, M. Preuss, J. Quinta da Fonseca, Texture memory and variant selection during phase transformation of a zirconium alloy, Acta Mater. 57 (2009) $5501-5511$ doi:10.1016/j.actamat.2009.07.046

[8] B. Roebuck, J. Lord, L. Orkney, Validation of a Miniature Tensile Strength Measurement System, in: Small Specim. Test Tech. Fourth Vol., ASTM International, 100 Barr Harbor Drive, PO Box C700, West Conshohocken, PA 19428-2959, n.d.: pp. 234-250. doi:10.1520/STP10824S.

[9] B. Roebuck, D.C. Cox, R.C. Reed, An Innovative Device for the Mechanical Testing of Miniature Specimens of Superalloys, in: Superalloys 2004 (Tenth Int. Symp., TMS, 2004 : pp. 523528. doi:10.7449/2004/Superalloys_2004_523_528.

[10] M. Newville, T. Stensitzki, D.B. Allen, A. Ingargiola, LMFIT: Non-Linear Least-Square Minimization and Curve-Fitting for Python, (2014). doi:10.5281/zenodo.11813.

[11] S. Matthies, G.W. Vinel, On the Reproduction of the Orientation Distribution Function of Texturized Samples from Reduced Pole Figures Using the Conception of a Conditional Ghost Correction, Phys. Status Solidi. 112 (1982) K111-K114. doi:10.1002/pssb.2221120254. 